Document downloaded from:

http://hdl.handle.net/10251/64680

This paper must be cited as:

Argyros, IK.; Cordero Barbero, A.; Magreñán, A.; Torregrosa Sánchez, JR. (2015). On the convergence of a damped Newton-like method with modified right hand side vector. Applied Mathematics and Computation. 266:927-936. doi:10.1016/j.amc.2015.05.148.

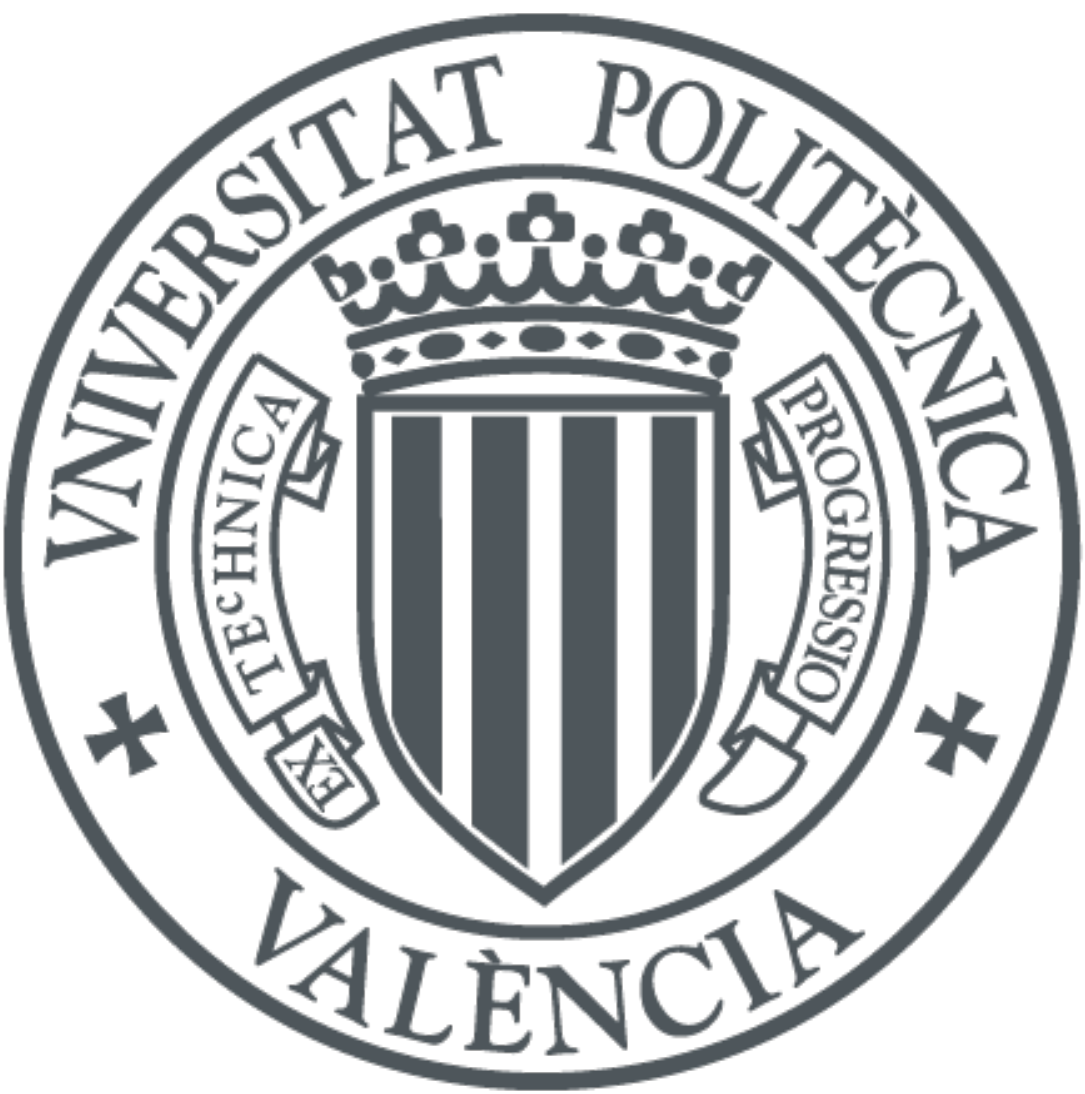

The final publication is available at

https://dx.doi.org/10.1016/j.amc.2015.05.148

Copyright Elsevier

Additional Information 


\title{
On the convergence of a damped Newton-like method with modified right hand side vector 光
}

\author{
Ioannis K. Argyros ${ }^{\mathrm{a}}$, Alicia Cordero ${ }^{\mathrm{b}}$, Alberto Magreñán ${ }^{\mathrm{c}}$, Juan R. Torregrosa ${ }^{\mathrm{b}, *}$ \\ ${ }^{a}$ Department of Mathematics Sciences, Cameron University, 73505 Lawton, OK, USA \\ ${ }^{b}$ Instituto Universitario de Matemática Multidisciplinar, Universitat Politècnica de València, 46022, València, Spain \\ ${ }^{c}$ Escuela de Ingeniería, Universidad Internacional de La Rioja, 26002 Logroño, La Rioja, Spain
}

\begin{abstract}
We present a convergence analysis for a Damped Newton-like method with modified right-hand side vector in order to approximate a locally unique solution of a nonlinear equation in a Banach spaces setting. In the special case when the method is defined on $\mathbb{R}^{m}$, our method provides computable error estimates based on the initial data. Such estimates were not given in relevant studies such as [1,2]. Numerical examples further validating the theoretical results are also presented in this study.
\end{abstract}

2010 Mathematics Subject Classification: 65H10, 65G99, 49M15.

Keywords: Damped Newton method, Banach space, local/semi-local convergence

\section{Introduction}

In this study we are concerned with the problem of approximating a locally unique solution $x^{*}$ of the nonlinear equation

$$
F(x)=0,
$$

where $F$ is a Fréchet-differentiable operator defined on a open convex subset $D$ of a Banach space $\mathbb{X}$ with values in a Banach space $\mathbb{Y}$.

Many problems from Computational Sciences and other disciplines can be brought in a form similar to equation (1) using Mathematical Modeling [3, 4, 5]. For example in data fitting, we have $\mathbb{X}=\mathbb{Y}=\mathbb{R}^{m}, m$ is the number of parameters and observations.

The solution of (1) can rarely be found in closed form. That is why the solution methods for these equations are usually iterative. In particular, the practice of Numerical Analysis for finding such solutions is essentially connected to Newton-like methods $[3,4,6,7]$. The study about convergence matter of iterative procedures is usually centered on two types: semilocal and local convergence analysis. The semilocal convergence matter is, based on the information around an initial point, to give criteria ensuring the convergence of iteration procedures; while the local one is, based on the information around a solution, to find estimates of the radii of the convergence balls.

In the present paper, we study the convergence of the Damped Newton-like method defined by

$$
x_{n+1}=x_{n}-\Lambda^{-1}\left(I-\alpha_{n}\left(A\left(x_{n}\right)-\Lambda\right)\right) F\left(x_{n}\right), \text { for each } n=0,1,2, \ldots,
$$

where $\Lambda \in \mathcal{L}(\mathbb{X}, \mathbb{Y})$ is the space of bounded linear operators from $\mathbb{X}$ into $\mathbb{Y}, \Lambda^{-1} \in \mathcal{L}(\mathbb{Y}, \mathbb{X}), A(x) \in \mathcal{L}(\mathbb{X}, \mathbb{Y})$ $(x \in D)$ is an approximation to $F^{\prime}(x), \alpha_{n}$ is a sequence of real numbers chosen to force convergence of sequence

\footnotetext{
仿 This research has been supported by Ministerio de Economía y Competitividad MTM2014-52016-C2-\{01,02 $\}$ and partially supported by the Universidad Internacional de La Rioja (UNIR, http://www.unir.net), under the Plan Propio de Investigación, Desarrollo e Innovación [2013-2015]. Research group of the third author: Matemática aplicada al mundo real (MAMUR)

${ }^{*}$ Corresponding author

Email addresses: iargyros@cameron.edu (Ioannis K. Argyros), acordero@mat.upv .es (Alicia Cordero), alberto.magrenan@unir.net (Alberto Magreñán), jrtorre@mat.upv.es (Juan R. Torregrosa)
} 
$\left\{x_{n}\right\}, x_{0}$ is an initial point and $I$ is the identity operator. If $\Lambda=F^{\prime}\left(x_{0}\right)$ and $\alpha_{n}=0$ for each $n=0,1,2, \ldots$, we obtain the modified Newton's method

$$
y_{n+1}=y_{n}-F^{\prime}\left(x_{0}\right)^{-1} F\left(y_{n}\right), y_{0}=x_{0}, \text { for each } n=0,1,2, \ldots,
$$

which converges linearly [3,5]. If $A(x)=F^{\prime}(x)$, Damped Newton-like method (2) reduces to

$$
v_{n+1}=v_{n}-\Lambda^{-1}\left(I-\alpha_{n}\left(F^{\prime}\left(v_{n}\right)-\Lambda\right)\right) F\left(v_{n}\right), \text { for each } n=0,1,2, \ldots,
$$

The local convergence of Damped Newton-like method (4) was studied by Krejić and Lužanin [2] (see also [1]) in the case when $\mathbb{X}=\mathbb{Y}=\mathbb{R}^{m}$.

Newton's method

$$
z_{n+1}=z_{n}-F^{\prime}\left(z_{n}\right)^{-1} F\left(z_{n}\right), \text { for each } n=0,1,2, \ldots,
$$

converges quadratically provided that the iteration starts close enough to the solution. However, the cost of a Newton iterate may be very expensive, since all the elements of the Jacobian matrix involved must be computed, as well as the need for an exact slowdown of a system of linear equations using a new matrix for every iterate. As noted in [2] Newton-like method (2) uses a modification of the right hand side vector, which is cheaper than the Newton and can be faster and more flexible than the modified Newton method. Notice also that modified Newton's method (3) is a special case of Newton's method (2). One step of the method requires the solution of a linear system, but the system matrix is the same in all iterations.

We present a new local and semilocal convergence analysis for Damped Newton-like method (2). In the local case the radius of convergence can be computed as well as the error bounds on the distances $\left\|x_{n}-x^{*}\right\|$ for each $n=0,1,2, \ldots$. In the semilocal case, we provide estimates on the smallness of $\left\|F\left(x_{0}\right)\right\|$ as well as computable estimates for $\left\|x_{n}-x^{*}\right\|$. Notice that such estimates were not given in [1, 2] for Damped Newton-like method (4).

We denote by $U(\nu, \mu)$ the open ball centered at $\nu \in \mathbb{X}$ and of radius $\mu>0$. Moreover, by $\overline{U(\nu, \mu)}$ we denote the closure of $U(\nu, \mu)$.

The paper is organized as follows. Sections 2 and 3 contain the semilocal and local convergence analysis of Newton-like method (2), respectively. The numerical examples are given in the concluding Section 4.

\section{Semilocal convergence}

In this section we present the semilocal convergence of Damped Newton-like method (2). We shall use the following conditions:

$\left(\mathrm{C}_{0}\right) F: D \subseteq \mathbb{X} \rightarrow \mathbb{Y}$ is Fréchet-differentiable and there exists $\Lambda \in \mathcal{L}(\mathbb{X}, \mathbb{Y})$ such that $\Lambda^{-1} \in \mathcal{L}(\mathbb{Y}, \mathbb{X})$ with $\left\|\Lambda^{-1}\right\| \leq a$

$\left(\mathrm{C}_{1}\right)$ There exists $L>0$ such that for each $x, y \in D$ the Lipschitz condition

$$
\left\|F^{\prime}(x)-F^{\prime}(y)\right\| \leq L\|x-y\|
$$

holds;

$\left(\mathrm{C}_{2}\right)$ There exist $L_{0}>0, a_{0} \geq 0$ such that for each $x \in D$ the center-Lipschitz condition

$$
\left\|F^{\prime}(x)-F^{\prime}\left(x_{0}\right)\right\| \leq L_{0}\left\|x-x_{0}\right\|
$$

and

$$
\left\|\Lambda-F^{\prime}\left(x_{0}\right)\right\| \leq a_{0}
$$

hold;

$\left(\mathrm{C}_{3}\right)$ There exist $x_{0} \in D, A(x) \in \mathcal{L}(\mathbb{X}, \mathbb{Y}),(x \in D), M \geq 0, \lambda \geq 0, \mu \geq 0, \lambda_{0} \geq 0$, such that for each $x \in D$

$$
\begin{gathered}
\left\|A(x)-A\left(x_{0}\right)\right\| \leq M\left\|x-x_{0}\right\|+\mu, \\
\left\|A\left(x_{0}\right)-\Lambda\right\| \leq \lambda
\end{gathered}
$$

and

$$
\left\|\Lambda^{-1}\left(A\left(x_{0}\right)-\Lambda\right)\right\| \leq \lambda_{0}
$$


$\left(\mathrm{C}_{4}\right)$ There exists $\alpha \geq 0$ such that

$$
\left|\alpha_{n}\right| \leq \alpha \text { for each } n=0,1,2, \ldots
$$

$\left(\mathrm{C}_{5}\right)$ There exist $q \in(0,1)$ and $x_{0} \in D$ such that

$$
\frac{L q^{2}}{2}\left\|F\left(x_{0}\right)\right\|+\left(\frac{L_{0} q}{1-q}\left\|F\left(x_{0}\right)\right\|+a_{0}\right) q+\alpha\left(\frac{M q\left\|F\left(x_{0}\right)\right\|}{1-q}+\lambda\right) \leq q ;
$$

and

$$
a+\alpha\left(\frac{a M q\left\|F\left(x_{0}\right)\right\|}{1-q}+a \mu+\lambda_{0}\right) \leq q
$$

hold;

$\left(\mathrm{C}_{6}\right)$ There exist $q \in(0,1) x_{0} \in D$ such that the second inequality in $\left(\mathrm{C}_{5}\right)$ and

$$
\left(\frac{2}{1-q}+\frac{1}{2}\right) L_{0} q\left\|F\left(x_{0}\right)\right\|+\left(\frac{L_{0} q}{1-q}\left\|F\left(x_{0}\right)\right\|+a_{0}\right) q+\alpha\left(\frac{M q\left\|F\left(x_{0}\right)\right\|}{1-q}+\lambda\right) \leq q
$$

hold;

$\left(\mathrm{C}_{7}\right) \overline{U\left(x_{0}, r\right)} \subseteq D$ with $r=\frac{q\left\|F\left(x_{0}\right)\right\|}{1-q}$.

Notice that $\left(\mathrm{C}_{1}\right)$ implies $\left(\mathrm{C}_{2}\right)$,

$$
L_{0} \leq L
$$

holds in general and $\frac{L}{L_{0}}$ can be arbitrarily large $[3,8,4]$. The conditions involving $\left\|F\left(x_{0}\right)\right\|$ and $q$ in $\left(\mathrm{C}_{5}\right)$ and $\left(\mathrm{C}_{6}\right)$ can be solved for $\left\|F\left(x_{0}\right)\right\|$ and $q$. However, these representations are very long and unattractive. That is why we decided to leave these conditions as uncluttered as possible. Notice also that these conditions determine the smallness of $\left\|F\left(x_{0}\right)\right\|$ and $q$. From now on we shall denote $\left(\mathbf{C}_{0}\right)-\left(\mathbf{C}_{5}\right),\left(\mathbf{C}_{7}\right)$ and $\left(\mathrm{C}_{0}\right),\left(\mathrm{C}_{2}\right)-\left(\mathrm{C}_{7}\right)$ as the $(C)$ and $\left(\mathbf{C}^{0}\right)$ conditions, respectively. Next, we present the semilocal convergence of the Damped Newton-like method (2) first under the (C) conditions.

Theorem 1. Suppose that the $(C)$ conditions hold. Then sequence $\left\{x_{n}\right\}$ generated by the Damped Newton-like method (2) is well defined, remains in $\overline{U\left(x_{0}, r\right)}$ for each $n=0,1,2, \ldots$, and converges to a solution $x^{*} \in \overline{U\left(x_{0}, r\right)}$ of equation (1). Moveover, the following estimates hold for each $n=0,1,2, \ldots$,

$$
\left\|x_{n+1}-x_{n}\right\| \leq q\left\|F\left(x_{n}\right)\right\| \leq q^{n+1}\left\|F\left(x_{0}\right)\right\|,
$$

and

$$
\left\|F\left(x_{n+1}\right)\right\| \leq q\left\|F\left(x_{n}\right)\right\| \leq q^{n+1}\left\|F\left(x_{0}\right)\right\|,
$$

where $q$ is defined in $\left(\mathrm{C}_{5}\right)$ and $r$ in $\left(\mathrm{C}_{7}\right)$.

Proof. We have by (2) and $\Lambda^{-1} \in \mathcal{L}(\mathbb{Y}, \mathbb{X})$ that sequence $\left\{x_{n}\right\}$ is well defined. Then, we shall show that $x_{1} \in U\left(x_{0}, r\right),\left\|x_{1}-x_{0}\right\| \leq q\left\|F\left(x_{0}\right)\right\|$ and $\left\|F\left(x_{1}\right)\right\| \leq q\left\|F\left(x_{0}\right)\right\|$. Indeed, we have by (2) for $n=0$ and $\left(\mathrm{C}_{0}\right)$, $\left(\mathrm{C}_{2}\right),\left(\mathrm{C}_{3}\right),\left(\mathrm{C}_{4}\right),\left(\mathrm{C}_{5}\right)$ and $\left(\mathrm{C}_{7}\right)$ that

$$
\begin{aligned}
\left\|x_{1}-x_{0}\right\| & =\left\|\Lambda^{-1}\left(I-\alpha_{0}\left(A\left(x_{0}\right)-\Lambda\right)\right) F\left(x_{0}\right)\right\| \\
& \leq\left[\left\|\Lambda^{-1}\right\|+\left|\alpha_{0}\right|\left\|\Lambda^{-1}\left(A\left(x_{0}\right)-\Lambda\right)\right\|\right]\left\|F\left(x_{0}\right)\right\| \\
& \leq\left[\left\|\Lambda^{-1}\right\|+\alpha \lambda_{0}\right]\left\|F\left(x_{0}\right)\right\| \\
& \leq q\left\|F\left(x_{0}\right)\right\|<r .
\end{aligned}
$$

Hence, $x_{1} \in U\left(x_{0}, r\right)$ and (13) holds for $n=0$. Using (2) and some algebraic manipulation, we obtain the Ostrowski-type approximation

$$
\begin{aligned}
F\left(x_{n+1}\right)= & \int_{0}^{1}\left[F^{\prime}\left(x_{n}+\theta\left(x_{n+1}-x_{n}\right)\right)-F^{\prime}\left(x_{n}\right)\right]\left(x_{n+1}-x_{n}\right) \mathrm{d} \theta \\
& +\left[\left(F^{\prime}\left(x_{n}\right)-F^{\prime}\left(x_{0}\right)\right)+\left(F^{\prime}\left(x_{0}\right)-\Lambda\right)\right]\left(x_{n+1}-x_{n}\right) \\
& +\alpha_{n}\left[\left(A\left(x_{n}\right)-A\left(x_{0}\right)\right)+\left(A\left(x_{0}\right)-\Lambda\right)\right] F\left(x_{n}\right) .
\end{aligned}
$$


Using (15), and the (C) conditions, for $n=0$ we get in turn that

$$
\begin{aligned}
\left\|F\left(x_{1}\right)\right\|= & \left\|\int_{0}^{1} F^{\prime}\left(x_{0}+\theta\left(x_{1}-x_{0}\right)\right)-F^{\prime}\left(x_{0}\right)\right\|\left\|x_{1}-x_{0}\right\| \mathrm{d} \theta \\
& +\left(\left\|F\left(x_{0}\right)-F^{\prime}\left(x_{0}\right)\right\|+\left\|F^{\prime}\left(x_{0}\right)-\Lambda\right\|\right)\left\|x_{1}-x_{0}\right\| \\
& +\left|\alpha_{0}\right|\left\|A\left(x_{0}\right)-\Lambda\right\|\left\|F\left(x_{0}\right)\right\| \\
\leq & \frac{L_{0}}{2}\left\|x_{1}-x_{0}\right\|^{2}+a_{0}\left\|x_{1}-x_{0}\right\|+\alpha \lambda\left\|F\left(x_{0}\right)\right\| \\
\leq & \frac{L}{2} q^{2}\left\|F\left(x_{0}\right)\right\|^{2}+a_{0} q\left\|F\left(x_{0}\right)\right\|+\alpha \lambda\left\|F\left(x_{0}\right)\right\| \\
\leq & \left(\frac{L}{2} q^{2}\left\|F\left(x_{0}\right)\right\|+a_{0} q+\alpha \lambda\right)\left\|F\left(x_{0}\right)\right\| \\
\leq & q\left\|F\left(x_{0}\right)\right\| .
\end{aligned}
$$

That is (14) holds for $n=0$. It follows from the existence of $x_{1} \in U\left(x_{0}, r\right)$ and $\Lambda^{-1} \in \mathcal{L}(\mathbb{X}, \mathbb{Y})$ that $x_{2}$ is well defined. Using (2) for $n=1$, we get by $\left(\mathrm{C}_{0}\right)-\left(\mathrm{C}_{5}\right)$ and $\left(\mathrm{C}_{7}\right)$ that

$$
\begin{aligned}
\left\|x_{2}-x_{1}\right\| & =\left\|\Lambda^{-1}\left(I-\alpha_{1}\left(A\left(x_{1}\right)-\Lambda\right)\right) F\left(x_{1}\right)\right\| \\
& \leq\left[\left\|\Lambda^{-1}\right\|+\left|\alpha_{1}\right|\left(\left\|\Lambda^{-1}\right\|\left\|\left(A\left(x_{1}\right)-A\left(x_{0}\right)\right)\right\|+\left\|\Lambda^{-1}\left(A\left(x_{0}\right)-\Lambda\right)\right\|\right)\right]\left\|F\left(x_{1}\right)\right\| \\
& \leq\left[a+\alpha\left(a M\left\|x_{1}-x_{0}\right\|+a \mu+\lambda_{0}\right)\right]\left\|F\left(x_{1}\right)\right\| \\
& \leq\left[a+\alpha\left(a M q\left\|F\left(x_{0}\right)\right\|+a \mu+\lambda_{0}\right)\right]\left\|F\left(x_{1}\right)\right\| \\
& \leq q\left\|F\left(x_{1}\right)\right\| \leq q^{2}\left\|F\left(x_{0}\right)\right\| .
\end{aligned}
$$

We also have that

$$
\begin{aligned}
\left\|x_{2}-x_{0}\right\| & \leq\left\|x_{2}-x_{1}\right\|+\left\|x_{1}-x_{0}\right\| \\
& \leq q^{2}\left\|F\left(x_{0}\right)\right\|+q\left\|F\left(x_{0}\right)\right\| \\
& =q\left\|F\left(x_{0}\right)\right\| \frac{1-q^{2}}{1-q}<\frac{q\left\|F\left(x_{0}\right)\right\|}{1-q}=r .
\end{aligned}
$$

That is, $x_{2} \in U\left(x_{0}, r\right)$. Then, using (15) for $n=1$, as above we get in turn that

$$
\begin{aligned}
\left\|F\left(x_{2}\right)\right\| \leq & \frac{L}{2}\left\|x_{2}-x_{1}\right\|^{2} \\
& +\left(\left\|F^{\prime}\left(x_{1}\right)-F^{\prime}\left(x_{0}\right)\right\|+\left\|F^{\prime}\left(x_{0}\right)-\Lambda\right\|\right)\left\|x_{2}-x_{1}\right\| \\
& +\left|\alpha_{1}\right|\left(\left\|A\left(x_{1}\right)-A\left(x_{0}\right)\right\|+\left\|A\left(x_{0}\right)-\Lambda\right\|\right)\left\|F\left(x_{1}\right)\right\| \\
\leq & \frac{L}{2} q^{2}\left\|F\left(x_{1}\right)\right\|^{2} \\
& +\left(L_{0}\left\|x_{1}-x_{0}\right\|+a_{0}\right) q\left\|F\left(x_{1}\right)\right\| \\
& +\alpha\left(M\left\|x_{1}-x_{0}\right\|+\mu+\lambda \|\right)\left\|F\left(x_{1}\right)\right\| \\
\leq & {\left[\frac{L}{2} q^{3}\left\|F\left(x_{0}\right)\right\|+\left(L_{0} q\left\|F\left(x_{0}\right)\right\|+a_{0}\right) q\right.} \\
& \left.+\alpha\left(M q\left\|F\left(x_{0}\right)\right\|+\mu+\lambda \|\right)\left\|F\left(x_{1}\right)\right\|\right] \\
\leq & q\left\|F\left(x_{1}\right)\right\| \leq q^{2}\left\|F\left(x_{0}\right)\right\| .
\end{aligned}
$$

Similarly, we have using (1.2) that

$$
\begin{aligned}
\left\|x_{3}-x_{2}\right\| & \leq\left[\left\|\Lambda^{-1}\right\|+\left|\alpha_{2}\right|\left(\left\|\Lambda^{-1}\left(A\left(x_{2}\right)-A\left(x_{0}\right)\right)\right\|+\left\|\Lambda\left(A\left(x_{0}\right)-\Lambda\right)\right\|\right)\right]\left\|F\left(x_{2}\right)\right\| \\
& \leq\left[a+\alpha\left(\left\|\Lambda^{-1}\right\|\left\|A\left(x_{2}\right)-A\left(x_{0}\right)\right\|+\lambda_{0}\right)\right]\left\|F\left(x_{2}\right)\right\| \\
& \leq\left[a+\alpha\left(a\left(M\left\|x_{2}-x_{0}\right\|+\mu\right)+\lambda_{0}\right)\right]\left\|F\left(x_{2}\right)\right\| \\
& \leq\left[a+\alpha\left(a M \frac{q\left\|F\left(x_{0}\right)\right\|}{1-q}+a \mu+\lambda_{0}\right)\right]\left\|F\left(x_{2}\right)\right\| \\
& \leq q\left\|F\left(x_{2}\right)\right\| \leq q^{3}\left\|F\left(x_{0}\right)\right\|
\end{aligned}
$$


where we used (2.3). We also have that

$$
\begin{aligned}
\left\|x_{3}-x_{0}\right\| & \leq\left\|x_{3}-x_{2}\right\|+\left\|x_{2}-x_{1}\right\|+\left\|x_{1}-x_{0}\right\| \\
& \leq\left(q^{3}+q^{2}+q\right)\left\|F\left(x_{0}\right)\right\| \\
& =q\left\|F\left(x_{0}\right)\right\| \frac{1-q^{3}}{1-q}<r .
\end{aligned}
$$

That is $x_{3} \in U\left(x_{0}, r\right)$. Moreover, using (2.10) as above we have that

$$
\begin{aligned}
\left\|F\left(x_{3}\right)\right\| \leq & \frac{L}{2}\left\|x_{3}-x_{2}\right\|^{2} \\
& +\left(\left\|F^{\prime}\left(x_{2}\right)-F^{\prime}\left(x_{0}\right)\right\|+\left\|F^{\prime}\left(x_{0}\right)-\Lambda\right\|\right)\left\|x_{3}-x_{2}\right\| \\
& +\left|\alpha_{3}\right|\left(\left\|A\left(x_{2}\right)-A\left(x_{0}\right)\right\|+\left\|A\left(x_{0}\right)-\Lambda\right\|\right)\left\|F\left(x_{2}\right)\right\| \\
\leq & \frac{L}{2} q^{2}\left\|F\left(x_{2}\right)\right\|^{2} \\
& +\left(L_{0}\left\|x_{2}-x_{0}\right\|+a_{0}\right) q\left\|F\left(x_{2}\right)\right\|+\alpha\left(M\left\|x_{2}-x_{0}\right\|+\lambda\right)\left\|F\left(x_{2}\right)\right\| \\
\leq & {\left[\frac{L}{2} q^{2}\left\|F\left(x_{2}\right)\right\|+\left(L_{0} \frac{q\left\|F\left(x_{0}\right)\right\|}{1-q}+a_{0}\right) q\right.} \\
+ & \left.\alpha\left(\frac{M q\left\|F\left(x_{0}\right)\right\|}{1-q}+\lambda\right)\right]\left\|F\left(x_{2}\right)\right\| \\
\leq & {\left[\frac{L}{2} q^{4}\left\|F\left(x_{0}\right)\right\|+\left(L_{0} \frac{q\left\|F\left(x_{0}\right)\right\|}{1-q}+a_{0}\right) q\right.} \\
+ & \left.\alpha\left(\frac{M q\left\|F\left(x_{0}\right)\right\|}{1-q}+\lambda\right)\right]\left\|F\left(x_{2}\right)\right\| \\
\leq & q\left\|F\left(x_{2}\right)\right\| \leq q^{3}\left\|F\left(x_{0}\right)\right\| .
\end{aligned}
$$

The rest follows in analogous way using induction (simply replace $x_{2}, x_{3}$ by $x_{n}, x_{n+1}$ in the preceding estimates). By letting $n \rightarrow \infty$ in (13) we obtain $F\left(x^{*}\right)=0$.

Condition $\left(\mathrm{C}_{1}\right)$ may not be satisfied but weaker condition $\left(\mathrm{C}_{2}\right)$ may be satisfied. In this case condition $\left(\mathrm{C}_{1}\right)$ can be dropped. Then, using instead of approximation (15) the approximation

$$
\begin{aligned}
F\left(x_{n+1}\right)= & \int_{0}^{1}\left[F^{\prime}\left(x_{n}+\theta\left(x_{n+1}-x_{0}\right)\right)-F^{\prime}\left(x_{0}\right)+\left(F^{\prime}\left(x_{0}\right)-F^{\prime}\left(x_{n}\right)\right)\right]\left(x_{n+1}-x_{n}\right) \mathrm{d} \theta \\
& +\left[\left(F^{\prime}\left(x_{n}\right)-F^{\prime}\left(x_{0}\right)\right)+\left(F^{\prime}\left(x_{0}\right)-\Lambda\right)\right]\left(x_{n+1}-x_{n}\right) \\
& +\alpha_{n}\left[\left(A\left(x_{n}\right)-A\left(x_{0}\right)\right)+\left(A\left(x_{0}\right)-\Lambda\right)\right] F\left(x_{n}\right),
\end{aligned}
$$

we arrive in an analogous way to Theorem 1 at the following semilocal convergence result for the Newton-like method (2) under the $\left(\mathrm{C}^{0}\right)$ conditions.

Theorem 2. Suppose that the $\left(C^{0}\right)$ conditions hold. Then sequence $\left\{x_{n}\right\}$ generated by the Damped Newton-like method (2) is well defined, remains in $\overline{U\left(x_{0}, r\right)}$ for each $n=0,1,2, \ldots$, and converges to a solution $x^{*} \in \overline{U\left(x_{0}, r\right)}$ of equation (1). Moveover, the following estimates hold for each $n=0,1,2, \ldots$,

$$
\left\|x_{n+1}-x_{n}\right\| \leq q\left\|F\left(x_{n}\right)\right\| \leq q^{n+1}\left\|F\left(x_{0}\right)\right\|,
$$

and

$$
\left\|F\left(x_{n+1}\right)\right\| \leq q\left\|F\left(x_{n}\right)\right\| \leq q^{n+1}\left\|F\left(x_{0}\right)\right\|,
$$

where $q$ is defined in $\left(\mathrm{C}_{6}\right)$ and $r$ in $\left(\mathrm{C}_{7}\right)$.

Concerning the uniqueness of the solution $x^{*}$ in $\overline{U\left(x_{0}, r\right)}$ we have the following result.

Proposition 1. Suppose that the $(C)$ or $\left(C^{0}\right)$ conditions hold. Moveover, suppose that there exist $x_{0} \in D$ and $r_{1} \geq r$ such that $F^{\prime}\left(x_{0}\right)^{-1} \in \mathcal{L}(\mathbb{Y}, \mathbb{X})$ and

$$
\left\|F^{\prime}\left(x_{0}\right)^{-1}\right\| L_{0}\left(r_{1}+r\right)<2 .
$$

Then the solution $x^{*}$ is the only solution of equation (1) in $\overline{U\left(x_{0}, r_{1}\right)}$, where $r$ is defined in $\left(\mathbf{C}_{7}\right)$. 
Proof. The existence of the solution $x^{*}$ is guaranteed by conditions $(\mathrm{C})$ or $\left(\mathrm{C}^{0}\right)$. To show uniqueness, let $y^{*} \in \overline{U\left(x_{0}, r_{1}\right)}$ with $F\left(y^{*}\right)=0$. Define $\mathcal{Q}=\int_{0}^{1} F^{\prime}\left(x^{*}+\theta\left(y^{*}-x^{*}\right)\right) \mathrm{d} \theta$. Then, using $\left(\mathrm{C}_{2}\right)$ and (18) we obtain in turn that

$$
\begin{aligned}
\left\|F^{\prime}\left(x_{0}\right)^{-1}\right\|\left\|\mathcal{Q}-F^{\prime}\left(x_{0}\right)\right\| & \leq\left\|F^{\prime}\left(x_{0}\right)^{-1}\right\| L_{0} \int_{0}^{1}\left\|\left(x^{*}-x_{0}\right)+\theta\left(y^{*}-x^{*}\right)\right\| \mathrm{d} \theta \\
& \leq\left\|F^{\prime}\left(x_{0}\right)^{-1}\right\| L_{0} \int_{0}^{1}\left\|(1-\theta)\left(x^{*}-x_{0}\right)+\theta\left(y^{*}-x_{0}\right)\right\| \mathrm{d} \theta \\
& \leq\left\|F^{\prime}\left(x_{0}\right)^{-1}\right\| \frac{L_{0}}{2}\left(r+r_{1}\right)<1 .
\end{aligned}
$$

It follows from (19) and the Banach lemma on invertible operator [5] that $\mathcal{Q}^{-1} \in \mathcal{L}(\mathbb{Y}, \mathbb{X})$. Moreover, we have that $0=F\left(y^{*}\right)-F\left(x^{*}\right)=\mathcal{Q}\left(y^{*}-x^{*}\right)$, which implies $x^{*}=y^{*}$.

\section{Local convergence}

In this section we present the local convergence of Damped Newton-like method (2). We shall use the following conditions:

$\left(\mathrm{H}_{0}\right) F: D \subseteq \mathbb{X} \rightarrow \mathbb{Y}$ is Fréchet-differentiable and there exists $\Lambda \in \mathcal{L}(\mathbb{X}, \mathbb{Y}), x^{*} \in D, \beta \geq 0, c \geq 0$ such that $\Lambda^{-1} \in \overline{\mathcal{L}}(\mathbb{Y}, \mathbb{X}), F\left(x^{*}\right)=0$ with $\left\|\Lambda^{-1}\right\| \leq a,\left\|F^{\prime}\left(x^{*}\right)\right\| \leq \beta$ and $\left\|\Lambda^{-1}\left(\Lambda-F^{\prime}\left(x^{*}\right)\right)\right\| \leq c$.

$\left(\mathrm{H}_{1}\right)$ There exists $L>0$ such that for each $x, y \in D$ the Lipschitz condition (6) holds;

$\left(\mathrm{H}_{2}\right)$ There exists $l_{0}>0$ such that for each $x \in D$ the center-Lipschitz condition

$$
\left\|F^{\prime}(x)-F^{\prime}\left(x^{*}\right)\right\| \leq l_{0}\left\|x-x^{*}\right\|
$$

holds;

$\left(\mathrm{H}_{3}\right)$ There exists $A(x) \in \mathcal{L}(\mathbb{X}, \mathbb{Y})(x \in D), M \geq 0, \mu \geq 0$ and $\gamma \geq 0$ such that for each $x \in D$

$$
\left\|A(x)-A\left(x^{*}\right)\right\| \leq M\left\|x-x^{*}\right\|+\mu
$$

and

$$
\left\|A(x)-A\left(x^{*}\right)\right\| \leq \gamma
$$

hold;

$\left(\mathrm{H}_{4}\right)\left|\alpha_{n}\right| \leq \alpha$

and

$$
c(1+\alpha \beta)+a(\mu+\gamma)<1 .
$$

Denote by $R_{1}$ the positive root of quadratic polynomial

$$
p_{1}(t):=\frac{\alpha a l_{0}^{2}}{2} t^{2}+\left(\frac{a L}{2}+\frac{c \alpha l_{0}}{2}+2 a l_{0}+a \alpha l_{0} \beta+a M\right) t+c(1+\alpha \beta)+a(\mu+\gamma)-1 .
$$

Moreover, denote by $R_{2}$ the positive root of quadratic polynomial

$$
p_{2}(t):=\frac{\alpha a l_{0}^{2}}{2} t^{2}+\left(\frac{3 a l_{0}}{2}+\frac{a c l_{0}}{2}+2 a l_{0}+\alpha \beta a l_{0}+a M\right) t+c(1+\alpha \beta)+a(\mu+\gamma)-1 ;
$$

$\left(\mathrm{H}_{5}\right) \overline{U\left(x^{*}, R\right)} \subseteq D$, where $R$ is $R_{1}$ or $R_{2}$. 
Notice that $\left(\mathrm{H}_{1}\right)$ implies $\left(\mathrm{H}_{2}\right)$,

$$
l_{0} \leq L
$$

holds in general and $\frac{L}{l_{0}}$ can be arbitrarily large $[3,4,8]$. The quadratic polynomials in $\left(\mathrm{H}_{4}\right)$ have a positive root by (20) and since the coefficients of $t$ and $t^{2}$ are positive. From now on we shall denote $\left(\mathrm{H}_{0}\right),\left(\mathrm{H}_{1}\right),\left(\mathrm{H}_{2}\right),\left(\mathrm{H}_{3}\right),\left(\mathrm{H}_{4}\right)$ and $\left(\mathrm{H}_{5}\right)$ and $\left(\mathrm{H}_{0}\right),\left(\mathrm{H}_{2}\right),\left(\mathrm{H}_{3}\right),\left(\mathrm{H}_{4}\right)$ and $\left(\mathrm{H}_{5}\right)$ as the $(\mathrm{H})$ and $\left(\mathrm{H}^{0}\right)$ conditions, respectively. Next, we present the local convergence of Damped Newton-like method (2) first under the $(\mathrm{H})$ conditions. In view of (2) and $F\left(x^{*}\right)=0$, we can have the following identity

$$
\begin{aligned}
x_{n+1}-x^{*}= & -\Lambda^{-1}\left\{\int_{0}^{1}\left[F^{\prime}\left(x^{*}+\theta\left(x_{n}-x^{*}\right)\right)-F^{\prime}\left(x_{n}\right)\right] \mathrm{d} \theta\right. \\
& -\left(\left(\Lambda-F^{\prime}\left(x^{*}\right)\right)+\left(F^{\prime}\left(x^{*}\right)-F^{\prime}\left(x_{n}\right)\right)\right)\left[\left(I-\alpha_{n} F^{\prime}\left(x^{*}\right)\right)\right. \\
& \left.-\alpha_{n} \int_{0}^{1}\left[F^{\prime}\left(x^{*}+\theta\left(x_{n}-x^{*}\right)\right)-F^{\prime}\left(x^{*}\right)\right]\right] \\
& \left.-\left(\left(A\left(x_{n}\right)-A\left(x^{*}\right)+\left(A\left(x^{*}\right)-F^{\prime}\left(x^{*}\right)\right)+\left(F^{\prime}\left(x^{*}\right)-F^{\prime}\left(x_{n}\right)\right)\right)\right)\right\}\left(x_{n}-x^{*}\right)
\end{aligned}
$$

Then, using (24), and the $(\mathrm{H})$ conditions, it is standard to arrive at $[3,4,5,6,7,8,9,10,11,12]$ :

Theorem 3. Suppose that the $(H)$ conditions hold. Then sequence $\left\{x_{n}\right\}$ generated by the Damped Newton method (2) is well defined, remains in $\overline{U\left(x^{*}, R_{1}\right)}$ for each $n=0,1,2, \ldots$, and converges to $x^{*}$ provided that $x_{0} \in$ $\overline{U\left(x^{*}, R_{1}\right)}$. Moveover, the following estimates hold for each $n=0,1,2, \ldots$,

$$
\left\|x_{n+1}-x^{*}\right\| \leq e_{n}\left\|x_{n}-x^{*}\right\|<\left\|x_{n}-x^{*}\right\|<R_{1},
$$

where

$$
\begin{aligned}
e_{n} & =\frac{L a}{2}\left\|x_{n}-x^{*}\right\|+\left(c+l_{0} a\left\|x_{n}-x^{*}\right\|\right) \times \\
& \left(1+\alpha \beta+\frac{\alpha l_{0}}{2}\left\|x_{n}-x^{*}\right\|+a\left(M\left\|x_{n}-x^{*}\right\|+\mu+\gamma+l_{0}\left\|x_{n}-x^{*}\right\|\right)\right) \\
& <p_{1}\left(R_{1}\right)+1<1 .
\end{aligned}
$$

In cases $\left(\mathrm{H}_{1}\right)$ cannot be verified by $\left(\mathrm{H}_{2}\right)$ holds, we can present the local convergence of the Damped Newton method (2) under the $\left(\mathrm{H}^{0}\right)$ conditions using the following modification of the Ostrowski representation (24) given by

$$
\begin{aligned}
x_{n+1}-x^{*}= & -\Lambda^{-1}\left\{\int_{0}^{1}\left[F^{\prime}\left(x^{*}+\theta\left(x_{n}-x^{*}\right)\right)-F^{\prime}\left(x^{*}\right)\right] \mathrm{d} \theta\right. \\
& +\left[F^{\prime}\left(x^{*}\right)-F^{\prime}\left(x_{n}\right)\right] \\
& -\left(\left(\Lambda-F^{\prime}\left(x^{*}\right)\right)+\left(F^{\prime}\left(x^{*}\right)-F^{\prime}\left(x_{n}\right)\right)\right)\left[\left(I-\alpha_{n} F^{\prime}\left(x^{*}\right)\right)\right. \\
& \left.-\alpha_{n} \int_{0}^{1}\left[F^{\prime}\left(x^{*}+\theta\left(x_{n}-x^{*}\right)\right)-F^{\prime}\left(x^{*}\right)\right]\right] \times \\
& \left.\left(\left(A\left(x_{n}\right)-A\left(x^{*}\right)+\left(A\left(x^{*}\right)-F^{\prime}\left(x^{*}\right)\right)+\left(F^{\prime}\left(x^{*}\right)-F^{\prime}\left(x_{n}\right)\right)\right)\right)\right\}\left(x_{n}-x^{*}\right)
\end{aligned}
$$

Theorem 4. Suppose that the $\left(H^{0}\right)$ conditions hold. Then sequence $\left\{x_{n}\right\}$ generated by the Damped Newton method (2) is well defined, remains in $\overline{U\left(x^{*}, R_{2}\right)}$ for each $n=0,1,2, \ldots$, and converges to $x^{*}$ provided that $x_{0} \in \overline{U\left(x^{*}, R_{2}\right)}$. Moveover, the following estimates hold for each $n=0,1,2, \ldots$,

$$
\left\|x_{n+1}-x^{*}\right\| \leq e_{n}^{0}\left\|x_{n}-x^{*}\right\|<\left\|x_{n}-x^{*}\right\|<R_{2},
$$

where

$$
\begin{aligned}
e_{n}^{0} & =\frac{3 l_{0} a}{2}\left\|x_{n}-x^{*}\right\|+\left(c+l_{0} a\left\|x_{n}-x^{*}\right\|\right)\left(1+\alpha \beta+\frac{\alpha l_{0}}{2}\left\|x_{n}-x^{*}\right\|\right) \\
& +a\left(M\left\|x_{n}-x^{*}\right\|+\mu+\gamma+l_{0}\left\|x_{n}-x^{*}\right\|\right) \\
& <p_{2}\left(R_{2}\right)+1<1 .
\end{aligned}
$$




\section{Numerical Examples}

In the following, we will present some numerical tests. These have been made by using software package Matlab 7.11.0 (R2011a) with Intel(R) Core(TM) i5-2500 CPU@3.30 GHz, RAM 16GB. For each example, we firstly analyze the conditions of semilocal and local convergence and then compare the numerical results obtained by different iterative schemes. In these tests, we use the approximated computational order of convergence ACOC, defined in [13] as

$$
A C O C=\frac{\ln \left(\left\|\mathbf{x}_{\mathbf{n}+\mathbf{1}}-\mathbf{x}_{\mathbf{n}}\right\| /\left\|\mathbf{x}_{\mathbf{n}}-\mathbf{x}_{\mathbf{n}-\mathbf{1}}\right\|\right)}{\ln \left(\left\|\mathbf{x}_{\mathbf{n}}-\mathbf{x}_{\mathbf{n}-\mathbf{1}}\right\| / \| \mathbf{x}_{\mathbf{n}-\mathbf{1}}-\mathbf{x}_{\mathbf{n}-\mathbf{2}} \mid\right)} .
$$

Example 1: Semilocal convergence. Let $X=Y=\mathcal{C}[0,1]$, the space of continuous functions defined in $[0,1]$ equipped with the max-norm. Let $\Omega=\{x \in \mathcal{C}[0,1] ;\|x\|<R\}$, such that $R>1$ and $F$ defined on $\Omega$ and given by

$$
F(x)(s)=x(s)-f(s)-\int_{0}^{1} G(s, t) x(t)^{3} d t, \quad x \in C[0,1], s \in[0,1],
$$

where $f \in \mathcal{C}[0,1]$ is a given function and the $\operatorname{kernel} G$ is the Green function

$$
G(s, t)= \begin{cases}(1-s) t, & t \leq s \\ s(1-t), & s \leq t\end{cases}
$$

In this case, for each $x \in \Omega, F^{\prime}(x)$ is a linear operator defined on $\Omega$ by the following expression:

$$
\left[F^{\prime}(x)(v)\right](s)=v(s)-3 \lambda \int_{0}^{1} G(s, t) x(t)^{2} v(t) d t, \quad v \in C[0,1], s \in[0,1] .
$$

Let us consider the particular case of the constant function $f(s)=1$. If we choose $x_{0}(s)=1$, it follows $\| I-$ $F^{\prime}\left(x_{0}\right) \| \leq 3 / 8$. Thus, $F^{\prime}\left(x_{0}\right)^{-1}$ is defined and

$$
\left\|F^{\prime}\left(x_{0}\right)^{-1}\right\| \leq \frac{8}{5} .
$$

Moreover,

$$
\left\|F\left(x_{0}\right)\right\| \leq \frac{1}{8}
$$

On the other hand, for $x, y \in \Omega$ we have

$$
\left[\left(F^{\prime}(x)-F^{\prime}(y)\right) v\right](s)=3 \int_{0}^{1} G(s, t)\left(x(t)^{2}-y^{2}(t)\right) v(t) d t .
$$

Consequently,

$$
\begin{gathered}
\left\|F^{\prime}(x)-F^{\prime}(y)\right\| \leq\|x-y\| \frac{3(\|x\|+\|y\|)}{8} \leq\|x-y\| \frac{6 R}{8}, \\
\left\|F^{\prime}(x)-F^{\prime}(1)\right\| \leq\|x-1\| \frac{1+3|\lambda|(\|x\|+1)}{8} \leq\|x-1\| \frac{4+3 R}{8} .
\end{gathered}
$$

Choosing $R=2.25, \alpha_{n}=\frac{1}{20}$ and $\Lambda=2$ and defining $A(x)=\frac{3}{8}$ we have that

$$
L=\frac{3}{2}, \quad L_{0}=1, \quad a=\frac{1}{2}, \quad M=\mu=0, \quad a_{0}=\frac{5}{8}, \quad \lambda=\frac{13}{8} \quad \text { and } \quad \lambda_{0}=\frac{13}{16} .
$$

Hence, for every $q \in(0.540625,0.59343)$ conditions (9) and (10) are satisfied. Moreover, considering $q=$ 0.55 condition $\left(\mathrm{C}_{7}\right)$ is satisfied with $r=\frac{q\left\|F\left(x_{0}\right)\right\|}{1-q}=0.152778 \ldots$ As a consequence, we can ensure the convergence of $\left\{x_{n}\right\}$ by Theorem 1 .

To find an estimated solution of the integral equation (28) we transform it into a finite dimensional problem by using a process of discretization. For this, we approximate the integral by the Gauss-Legendre formula

$$
\int_{0}^{1} h(t) d t \approx \sum_{i=1}^{m} w_{i} h\left(t_{i}\right)
$$


where the nodes $t_{i}$ and the weights $w_{i}$ are known.

If we denote the approximation of $x\left(t_{i}\right)$ by $x_{i}(i=1,2, \ldots, m)$, then (28) is transformed into the following nonlinear system of equations

$$
x_{i}=1+\sum_{j=1}^{m} a_{i j} x_{j}^{3}, \quad i=1,2, \ldots, m,
$$

where

$$
a_{i j}= \begin{cases}w_{j} t_{j}\left(1-t_{i}\right) & \text { if } j \leq i, \\ w_{j} t_{i}\left(1-t_{j}\right) & \text { if } j>i .\end{cases}
$$

System (29) is now written as

$$
H(x)=x-\overline{1}-A v_{x}=0, \quad H: \mathbb{R}^{m} \rightarrow \mathbb{R}^{m},
$$

where

$$
x=\left(x_{1}, x_{2}, \ldots, x_{m}\right)^{T}, \overline{1}=(1,1, \ldots, 1)^{T}, A=\left(a_{i j}\right)_{i, j=1}^{m}, \text { and } v_{x}=\left(x_{1}^{3}, x_{2}^{3}, \ldots, x_{m}^{3}\right)^{T} .
$$

Moreover, $H^{\prime}(x)=I_{m}-3 A D(x)$, where $D(x)=\operatorname{diag}\left\{x_{1}^{2}, x_{2}^{2}, \ldots, x_{m}^{2}\right\}$ and $I_{m}$ denotes the identity matrix of size $m \times m$.

In order to apply Theorem 1 with $x_{0}=(1,1, \ldots, 1)^{T}$ and $m=8$ it is necessary to calculate the involved constants. It is easy to check that

$$
\left\|H^{\prime}(x)-H^{\prime}(y)\right\| \leq 6\|A\|\|x-y\|
$$

and

$$
\left\|H^{\prime}(x)-H^{\prime}\left(x_{0}\right)\right\| \leq 6\|A\|\left\|x-x_{0}\right\| .
$$

So, $L=L_{0}=0.627$ and $\left\|F\left(x_{0}\right)\right\|=0.2285$. Now, we choose $\Lambda=A(x)=I_{8}+3 A$; then,

$$
a=0.6154, \quad M=\mu=0, \quad a_{0}=0.6264, \quad \lambda=\lambda_{0}=0
$$

and for every $q \in(0.6154,0.6936)$, conditions (C) are satisfied. By considering $q=0.62$, we obtain $r=0.3728$ as the convergence radius. Under these assumptions, the proposed Damped Newton method has the following iterative expression:

$$
x_{n+1}=x_{n}-\left(I_{8}+3 A\right)^{-1} H\left(x_{n}\right),
$$

that will be denoted by DN1.

In the following, we will present some numerical tests. These have been made by using the stopping criterium $\left\|F\left(x_{n+1}\right)\right\|<10^{-9}$ or $\left\|\mathbf{x}_{\mathbf{n}+\mathbf{1}}-\mathbf{x}_{\mathbf{n}}\right\|<10^{-9}$. In Table 1 , we show the numerical results obtained for this problem, with different initial estimations and by applying iterative methods DN1, Newton's scheme $\mathrm{N}$ and also Modified Newton MN. We show, the number of iterations, the approximated computational order of convergence ACOC, the difference between the last iteration and the preceding one $\left\|\mathbf{x}_{\mathbf{n}+\mathbf{1}}-\mathbf{x}_{\mathbf{n}}\right\|$, the residual of the function at the last iteration, $\left\|F\left(\mathbf{x}_{\mathbf{n}+\mathbf{1}}\right)\right\|$ and the mean elapsed time (CPU-time) after 500 executions.

In all cases, every method has converged to the solution

$$
(1.0147 \ldots, 1.0711 \ldots, 1.1460 \ldots, 1.2000 \ldots, 1.2000 \ldots, 1.1460 \ldots, 1.0711 \ldots, 1.0147 \ldots)^{T},
$$

except in the third case for Newton's method, that has converged to another solution.

Let us observe that the lower number of iterations of method DN1 corresponds to the initial estimation $(1, \ldots, 1)^{T}$; it is clear as the iterative expression has been built for this specific initial guess. However, for another initial approximations, the behavior of the method can be even better than the one of Modified Newton's scheme.

Example 2: Local convergence. To solve the equation of molecular interaction, (see [14])

$$
\begin{array}{rc}
u_{x x}+u_{y y}=u^{2}, & (x, y) \in[0,1] \times[0,1], \\
u(x, 0)=2 x^{2}-x+1, & u(x, 1)=2, \\
u(0, y)=2 y^{2}-y+1, & u(1, y)=2,
\end{array}
$$


we need to deal with a boundary value problem with a nonlinear partial differential equation of second order. To estimate its solution numerically, we have used central divided differences in order to transform the problem in a nonlinear system of equations, which is solved by using the proposed method.

The discretization process yields to the nonlinear system of equations,

$$
u_{i+1, j}-4 u_{i, j}+u_{i-1, j}+u_{i, j+1}+u_{i, j-1}-h^{2} u_{i, j}^{2}=0 i=1, \ldots, n x, j=1, \ldots, n y,
$$

where $u_{i, j}$ denotes the estimation of the unknown $u\left(x_{i}, y_{j}\right), x_{i}=i h$ with $i=0,1, \ldots, n x, y_{j}=j k$ with $j=0,1, \ldots, n y$, are the nodes in both variables, being $h=\frac{1}{n x}, k=\frac{1}{n y}$ and $n x=n y$.

In this case, we fix $n x=n y=4$, so a mesh of $5 \times 5$ is generated. As the boundary conditions give us the value of the unknown function at the nodes $\left(x_{0}, y_{j}\right),\left(x_{4}, y_{j}\right)$ for all $j$ and also at $\left(x_{i}, y_{0}\right),\left(x_{i}, y_{4}\right)$ for all $i$, we have only nine unknowns, that are renamed as:

$$
x_{1}=u_{1,1}, \quad x_{2}=u_{2,1}, \quad x_{3}=u_{3,1}, x_{4}=u_{1,2}, \quad x_{5}=u_{2,2}, \quad x_{6}=u_{3,2}, x_{7}=u_{1,3}, \quad x_{8}=u_{2,3}, x_{9}=u_{3,3} .
$$

So, for $X=Y=\mathbb{R}^{9}$ and $D=\mathbb{R}^{9}$, the system can be expressed as

$$
F(x)=A x+\phi(x)-b=0,
$$

where

$$
A=\left(\begin{array}{ccc}
M & -I_{3} & 0 \\
-I_{3} & M & -I_{3} \\
0 & -I_{3} & M
\end{array}\right), \quad \text { being } \quad M=\left(\begin{array}{ccc}
4 & -1 & 0 \\
-1 & 4 & -1 \\
0 & -1 & 4
\end{array}\right), \quad \phi(x)=h^{2}\left(x_{1}^{2}, x_{2}^{2}, \ldots, x_{9}^{2}\right)^{T},
$$

$I_{3}$ is the $3 \times 3$ identity matrix and $b=\left(\frac{7}{4}, 1, \frac{27}{8}, 1,0,2, \frac{27}{8}, 2,4\right)^{T}$. In this case,

$$
F^{\prime}(x)=A+2 h^{2} \operatorname{diag}\left(x_{1}, \ldots, x_{9}\right)
$$

and the solution of the problem is in Table 2, with 15 exact digits.

Now, we consider $\Lambda=7 I_{9}, A(x)=A$ and $\alpha_{n}=10^{-3}$, for all $n \geq 0$. It can be checked that, by using $\alpha=10^{-3}, \beta=7.007, L=l_{0}=\frac{1}{8}, M=\mu=\gamma=0, c=0.81$ and $a=\frac{1}{7}$, conditions (H) are fulfilled. On the other hand, the positive root of polynomial $p_{1}(t)$ is $R_{1}=4.11225$. So, convergence is assured by Theorem 3 (the same values allow us to apply Theorem 4 , obtaining the same radius of convergence). Under these assumption, the iterative expression of the proposed method, that will be denoted by DN2, is

$$
x_{n+1}=x_{n}-\frac{1}{7}\left(I_{9}-10^{-3}\left(A-7 I_{9}\right)\right) F\left(x_{n}\right) .
$$

Now, we will check the performance of the methods by means of some numerical tests. These tests have been made by using the stopping criterium $\left\|F\left(x_{n+1}\right)\right\|<10^{-9}$ or $\left\|x_{n+1}-x_{n}\right\|<10^{-9}$. In Table 3 , we show the numerical results obtained for the problem of molecular interaction (32), with different initial estimations and by applying the iterative methods DN2. We show the number of iterations, the approximated computational order of convergence ACOC, the difference between the last iteration and the preceding one $\left\|x_{n+1}-x_{n}\right\|$, the residual of the function at the last iteration, $\left\|F\left(x_{n+1}\right)\right\|$ and the mean elapsed time after 500 executions.

As we can see in Table 3, although the convergence is only linear we can choose initial estimations far from the solution, with assured convergence. In this case, we have not included in Table 3 a comparison with Newton's method and Modified Newton's scheme, because these methods are always clearly better than DN2 in terms of number of iterations.

Example 3: Local convergence. Let $X=Y=\mathbb{R}^{3}, D=U(0,1)$ and $x^{*}=(0,0,0)$. Define function $F$ on $D$ for $w=(x, y, z)$ by

$$
F(w)=\left(e^{x}-1, \frac{e-1}{2} y^{2}+y, x+z\right) .
$$

Then, the Fréchet derivative of $F$ is given by

$$
F^{\prime}(w)=\left(\begin{array}{ccc}
e^{x} & 0 & 0 \\
0 & (e-1) y+1 & 0 \\
1 & 0 & 1
\end{array}\right)
$$


Notice that we have $F\left(x^{*}\right)=0$,

$$
F^{\prime}\left(x^{*}\right)=\left(\begin{array}{lll}
1 & 0 & 0 \\
0 & 1 & 0 \\
1 & 0 & 1
\end{array}\right), \quad \text { and } \quad F^{\prime}\left(x^{*}\right)^{-1}=\left(\begin{array}{ccc}
1 & 0 & 0 \\
0 & 1 & 0 \\
-1 & 0 & 1
\end{array}\right)
$$

We take $\Lambda=F^{\prime}\left(x^{*}\right), A(x)=e I_{3}$ and $\alpha_{n}=10^{-3}$. Computing we obtain $l_{0}=e-1<L=e, a=1.62$, $\beta=1.62, c=0, M=0, \mu=0$ and $\gamma=0$. So the $(H)$ conditions are satisfied and Theorem 3 ensures the convergence of the method 2 in $\overline{U\left(x^{*}, R_{1}\right)}$, being $R_{1}=0.1286 \ldots$. The iterative expression of the resulting scheme, will be denoted by DN3.

In the following, we will test DN3, N and MD methods by using the stopping criterium $\left\|F\left(w_{n+1}\right)\right\|<10^{-12}$ or $\left\|w_{n+1}-w_{n}\right\|<10^{-12}$. In Table 4 , we show the number of iterations, the approximated computational order of convergence ACOC, the difference between the last iteration and the preceding one $\left\|w_{n+1}-w_{n}\right\|$, the residual of the function at the last iteration, $\left\|F\left(w_{n+1}\right)\right\|$ and the the mean elapsed time after 500 executions.

Let us remark that the mean elapsed time of DN3 is, in the first and second cases, better than the one of Newton's method. In all cases, DN3 has a better performance than Modified Newton's scheme.

\section{Acknowledgements}

The authors thank to the anonymous referees for their valuable comments and suggestions.

\section{References}

[1] D. Herceg, N. Krejić and Z. Lužanin, Quasi-Newton's method with correction, Novi Sad J. Math. 26 (1996) 115-127.

[2] N. Krejić and Z. Lužanin, Newton-like method with modification of the right-hand-side vector, Math. Comp. 71 (2002) 237-250.

[3] I.K. Argyros, Convergence and applications of Newton-type iterations, Springer-Verlag Publ., New York, 2008.

[4] I.K. Argyros and S. Hilout, Computational methods in Nonlinear Analysis, World Scientific Publ. Comp. New Jersey, 2013.

[5] L.V. Kantorovich and G.P. Akilov, Functional Analysis, Pergamon Press, Oxford, 1982.

[6] I. K. Argyros and J. Chen, On local convergence of a Newton-type method in Banach space, Int. J. Comput. Math. 86 (2009) 1366-1374.

[7] I.K. Argyros and S. Hilout, Improved local convergence of Newton's method under weak majorant condition, J. Comput. Appl. Math. 236 (2012) 1892-1902.

[8] I. K. Argyros and J. Chen, Improved results on estimating and extending the radius of an attraction ball, Appl. Math. Lett. 23 (2010) 404-408.

[9] J. Chen and Q. Sun, The convergence ball of Newton-like methods in Banach space and applications, Taiwanese J. Math. 11 (2007) 383-397.

[10] J. Chen and W. Li, Convergence behaviour of inexact Newton methods under weak Lipschitz condition, J. Comput. Appl. Math. 191 (2006) 143-164.

[11] F.A. Potra, Sharp error bounds for a class of Newton-like methods, Libertas Mathematica, 5 (1985) 71-84.

[12] P.D. Proinov, General local convergence theory for a class of iterative processes and its applications to Newton's method, J. Complex. 25 (2009) 38-62.

[13] A. Cordero and J.R. Torregrosa, Variants of Newton's method using fifth-order quadrature formulas, Appl. Math. Comp. 190 (2007) 686-698.

[14] L.B. Rall, Computational Solution of Nonlinear Operator Equations, Robert E. Krieger Publishing Company, Inc., 1969. 


\begin{tabular}{|c|c|c|c|c|c|c|}
\hline \hline Method & $x_{0}$ & iter & ACOC & $\left\|x_{n+1}-x_{n}\right\|$ & $\left\|F\left(x_{n+1}\right)\right\|$ & e-time \\
\hline DN1 & $(1, \ldots, 1)^{T}$ & 34 & 1.0000 & $9.53 \mathrm{e}-10$ & $7.09 \mathrm{e}-10$ & 0.0145 \\
N & $(1, \ldots, 1)^{T}$ & 4 & 1.9972 & $2.78 \mathrm{e}-8$ & $1.81 \mathrm{e}-16$ & 0.0017 \\
MN & $(1, \ldots, 1)^{T}$ & 11 & 1.0000 & $3.79 \mathrm{e}-9$ & $4.78 \mathrm{e}-10$ & 0.0042 \\
\hline DN1 & $(1.5, \ldots, 1.5)^{T}$ & 36 & 1.0000 & $1.19 \mathrm{e}-9$ & $8.82 \mathrm{e}-10$ & 0.0149 \\
N & $(1.5, \ldots, 1.5)^{T}$ & 5 & 1.9972 & $2.78 \mathrm{e}-8$ & $1.81 \mathrm{e}-16$ & 0.0029 \\
MN & $(1.5, \ldots, 1.5)^{T}$ & 178 & 1.0000 & $3.67 \mathrm{e}-9$ & $9.88 \mathrm{e}-10$ & 0.0709 \\
\hline DN1 & $(2, \ldots, 2)^{T}$ & 40 & 1.0000 & $1.2 \mathrm{e}-9$ & $8.89 \mathrm{e}-10$ & 0.0163 \\
N & $(2, \ldots, 2)^{T}$ & 6 & 2.0007 & $1.98 \mathrm{e}-7$ & $1.47 \mathrm{e}-14$ & 0.0023 \\
MN & $(2, \ldots, 2)^{T}$ & $>10^{4}$ & - & - & - & - \\
\hline \hline
\end{tabular}

Table 1: Numerical tests for different initial estimations in Example 1 


\begin{tabular}{|c|c|}
\hline \hline & $x^{*}$ \\
\hline$u_{1,1}$ & $1.025911711690039 \ldots$ \\
$u_{2,1}$ & $1.209713887135790 \ldots$ \\
$u_{3,1}$ & $1.516703030959235 \ldots$ \\
$u_{1,2}$ & $1.209713887135790 \ldots$ \\
$u_{2,2}$ & $1.387703786439461 \ldots$ \\
$u_{3,2}$ & $1.625872491958707 \ldots$ \\
$u_{1,3}$ & $1.516703030959235 \ldots$ \\
$u_{2,3}$ & $1.625872491958707 \ldots$ \\
$u_{3,3}$ & $1.764299485442884 \ldots$ \\
\hline \hline
\end{tabular}

Table 2: Approximated solution 


\begin{tabular}{|c|c|c|c|c|c|}
\hline \hline$x_{0}$ & iter & ACOC & $\left\|x_{n+1}-x_{n}\right\|$ & $\left\|F\left(x_{n+1}\right)\right\|$ & e-time \\
\hline$(0.5, \ldots, 0.5)^{T}$ & 95 & 1.0000 & $8.86 \mathrm{e}-10$ & $4.97 \mathrm{e}-9$ & 0.0032 \\
$(1, \ldots, 1)^{T}$ & 91 & 1.0000 & $9.32 \mathrm{e}-10$ & $5.23 \mathrm{e}-9$ & 0.0031 \\
$(1.5, \ldots, 1.5)^{T}$ & 83 & 1.0000 & $9.28 \mathrm{e}-10$ & $5.21 \mathrm{e}-9$ & 0.0028 \\
$(2, \ldots, 2)^{T}$ & 92 & 1.0000 & $9.67 \mathrm{e}-10$ & $5.43 \mathrm{e}-9$ & 0.0031 \\
$(2.5, \ldots, 2.5)^{T}$ & 95 & 1.0000 & $9.19 \mathrm{e}-10$ & $5.16 \mathrm{e}-9$ & 0.0032 \\
$(2.7, \ldots, 2.7)^{T}$ & 96 & 1.0000 & $8.69 \mathrm{e}-10$ & $4.87 \mathrm{e}-9$ & 0.0033 \\
\hline \hline
\end{tabular}

Table 3: Numerical tests for different initial estimations in Example 3 


\begin{tabular}{|c|c|c|c|c|c|c|}
\hline \hline Method & $w_{0}$ & iter & ACOC & $\left\|w_{n+1}-w_{n}\right\|$ & $\left\|F\left(w_{n+1}\right)\right\|$ & e-time \\
\hline DN3 & $(0.06,0.06,0.05)^{T}$ & 6 & 1.0267 & $4.77 \mathrm{e}-11$ & $1.05 \mathrm{e}-13$ & $1.56 \mathrm{e}-4$ \\
N & $(0.06,0.06,0.05)^{T}$ & 4 & 1.9310 & $3.89 \mathrm{e}-11$ & $1.11 \mathrm{e}-16$ & $1.87 \mathrm{e}-4$ \\
MN & $(0.06,0.06,0.05)^{T}$ & 11 & 0.9999 & $1.35 \mathrm{e}-12$ & $1.39 \mathrm{e}-13$ & $2.5 \mathrm{e}-4$ \\
\hline DN3 & $(0,0.12,0)^{T}$ & 5 & 1.0113 & $4.66 \mathrm{e}-10$ & $8.03 \mathrm{e}-13$ & $1.56 \mathrm{e}-4$ \\
N & $(0,0.12,0)^{T}$ & 4 & 1.9999 & $6.78 \mathrm{e}-9$ & $3.95 \mathrm{e}-17$ & $1.87 \mathrm{e}-4$ \\
MN & $(0,0.12,0)^{T}$ & 15 & 1.0000 & $8.59 \mathrm{e}-13$ & $1.77 \mathrm{e}-13$ & $2.5 \mathrm{e}-4$ \\
\hline DN3 & $(-0.05,0.02,-0.06)^{T}$ & 6 & 1.0236 & $3.46 \mathrm{e}-11$ & $7.53 \mathrm{e}-14$ & $2.18 \mathrm{e}-4$ \\
N & $(-0.05,0.02,-0.06)^{T}$ & 3 & 1.9486 & $1.15 \mathrm{e}-6$ & $3.26 \mathrm{e}-13$ & $9.36 \mathrm{e}-5$ \\
MN & $(-0.05,0.02,-0.06)^{T}$ & 9 & 1.0000 & $1.78 \mathrm{e}-12$ & $6.15 \mathrm{e}-14$ & $2.5 \mathrm{e}-4$ \\
\hline \hline
\end{tabular}

Table 4: Numerical tests for different initial estimations in Example 3 